Pacific Journal of Mathematic 


\section{CONJUGATE SERIES AND A THEOREM OF PALEY}

\section{HENRY HELSON}

1. Introduction. It is known that a trigonometric series

$$
\sum_{-\infty}^{\infty} a_{n} e^{i n x}
$$

does not have to satisfy condition on the size of its coefficients stronger than the trivial one

$$
\sum_{-\infty}^{\infty}\left|a_{n}\right|^{2}<\infty
$$

in order to be the Fourier series of a continuous function. One theorem which gives precise content to this general statement is the following: If $\left\{w_{n}\right\}_{-\infty}^{\infty}$ is a sequence of non-negative numbers such that

$$
\sum_{-\infty}^{\infty}\left|a_{n}\right| w_{n}<\infty
$$

whenever (1) is the Fourier series of a continuous function, then

$$
\sum_{-\infty}^{\infty} w_{n}^{2}<\infty .
$$

The fact that (1) is the Fourier series of a continuous function does not by any means imply the same for

$$
\sum_{0}^{\infty} a_{n} e^{i n x}
$$

Therefore the following rather neglected theorem of Paley [5] lies deeper than the result just stated.

THEOREM 1 (Paley). If $\left\{w_{n}\right\}_{0}^{\infty}$ is a sequence of non-negative numbers such that

$$
\sum_{0}^{\infty}\left|a_{n}\right| w_{n}<\infty
$$

whenever (2) is the Fourier series of a continuous function, then

$$
\sum_{0}^{\infty} w_{n}^{2}<\infty .
$$

In the next section we offer a new and simple proof of this theorem. The proof depends on the fact that the conjugate series of a Fourier-

Received April 14, 1958. The author is a fellow of the Alfred P. Sloan foundation. 
Stieltjes series is summable (by Abel or Cesàro means) almost everywhere.

In several or infinitely many variables the analogue of the first result mentioned is true. The main purpose of this note is to investigate a few of the possible generalizations of Paley's theorem to functions of several variables.

In $\S 3$ we develop the notion of conjugate function in several variables from a point of view somewhat different from that of other writers. We cannot answer the natural questions about the summability of conjugate series, but our result makes it possible (in $\S 4$ ) to apply a theorem of Zygmund on the summability of multiple power series in order to generalize Paley's Theorem.

Finally, in the last section, we show by a simple example that Paley's theorem cannot be extended to power series in infinitely many variables. We do not know, however, whether power series in two variables have Paley's property or not.

2. Proof of Paley's theorem. For each continuous function $\varphi$ with Fourier series (2) define

$$
F[\varphi]=\sum_{0}^{\infty} a_{n} w_{n} .
$$

The series converges on account of the hypothesis (3), and evidently $F$ is a continuous linear functional defined on a closed subspace of the space $C$ of continuous periodic functions. Extend $F$ to a linear functional on all of $C$. By a well-known theorem of F. Riesz, there is a complex bounded measure $\mu$ defined on Borel subsets of the circle such that

$$
F[\psi]=\int_{0}^{2 \pi} \psi(-x) d \mu(x)
$$

In particular, for the functions $\psi(x)=e^{i n x}(n=0,1,2, \cdots)$ we have two representations for $F[\psi]$ given by (5) and (6) :

$$
w_{n}=F[\psi]=\int_{0}^{2 \pi} e^{-i n x} d \mu(x) .
$$

If we define $w_{n}$ by (7) for $n<0$, the Fourier-Stieltjes series of $d \mu$ is

$$
\sum_{-\infty}^{\infty} w_{n} e^{i n x} .
$$

Now every Fourier-Stieltjes series is summable almost everywhere by Abel (or by Cesàro) means [7, p. 59]. The same is true, although more difficult to prove, for the conjugate series of a Fourier-Stieltjes series [7, p. 146]. It follows from these facts together that 


$$
\sum^{\infty} w_{n} e^{i n x}
$$

is summable almost everywhere.

Instead of (5) we could have defined $F$ as

$$
F[\varphi]=\sum_{0}^{\infty} a_{n} w_{n} e^{i t_{n}},
$$

where $t_{0}, t_{1}, \cdots$ are any real numbers. The same argument shows that

$$
\sum_{0}^{\infty} w_{n} e^{i t_{n}} e^{i n x}
$$

is summable for almost all $x$, no matter what values the $t_{n}$ have. But this implies (4) [7, p. 125], as was to be proved.

3. Conjugate series. Denote by $T_{k}$ the torus group in $k$ dimensions, and by $T$ the compact infinite-dimensional torus. The dual of $T_{k}$ is the lattice group $\mathscr{L}_{k}$ in $k$ dimensions. The dual $\mathscr{L}$ of $T$ is the group whose elements are sequences of integers $\left(n_{1}, n_{2}, \cdots\right)$ which are all zero but a finite number. A summable function $f$ on $T_{k}$ has Fourier series

$$
f\left(x_{1}, \cdots, x_{k}\right) \sim \sum a\left(n_{1}, \cdots, n_{k}\right) e^{i\left(n_{1} x_{1}+\cdots+n_{k} x_{k}\right)} .
$$

(The formal summation is extended over all integral values of $n_{1}, \cdots, n_{k}$.) Similarly we write for a summable $f$ on $T$

$$
f\left(x_{1}, \cdots\right) \sim \sum a\left(n_{1}, \cdots\right) e^{i\left(n_{1} x_{1}+\cdots\right)},
$$

where the sum in the exponent is actually finite at each lattice point $\left(n_{1}, \cdots\right)$.

Let $N$ and $X$ denote generic points of $\mathscr{L}_{k}$ (or of $\mathscr{L}$ ) and of $T_{k}$ (or of $T$ ) respectively. In place of (8) and (9) we shall write

$$
f(X) \sim \sum a(N) e^{i N \cdot X}
$$

In the same way we write the Fourier-Stieltjes series of a bounded complex measure $\mu$ on $T_{k}$ or on $T$

$$
d \mu(X) \sim \sum a(N) e^{i_{N} \cdot X}
$$

Let $S$ be a subset of $\mathscr{L}_{k}$ or of $\mathscr{L}$; we are interested in the operator $T_{S}$ which carries every series (10) into

$$
\sum_{S} a(N) e^{i N \cdot x} .
$$

If $T_{S}$ carries the Fourier series of one function space into those of another, we shall consider $T_{S}$ at the same time as an operation on functions. Our aim is to prove, by specializing $S$, that $T_{S}$ is continuous in certain topologies. 
Definition. A subset $S$ of $\mathscr{L}_{k}$ or of $\mathscr{L}$ is a half-space if $1^{\circ}$ The origin is not in $S$

$2^{\circ} \quad N \in S$ if and only if $-N \notin S$ ( $N$ not the origin)

$3^{\circ} M+N$ belongs to $S$ with $M$ and $N$.

Fourier series with coefficients lying in a half-space have many of the properties of analytic Fourier series of one variable [4]. The following theorem of Bochner [1] generalizes a well-known theorem of M. Riesz.

Bochner's THEOREM. If $S$ is a half-space, then $T_{S}$ is a bounded operator mapping $L^{p}$ into itself for each $p>1$.

The main result of this section is of the same character.

THEOREM 2. If $S$ is a half-space and $f$ is a trigonometric polynomial then

$$
\left\|T_{S} F\right\|_{p} \leqq K_{p}\|f\|_{1}
$$

where $K_{p}$ is a constant depending only on $p$.

The proof is like that of the corresponding theorem in one variable [7, p. 150], applied not to analytic functions but to elements in an appropriate Banach algebra. The observations which follow are not new.

Denote by $C_{S}$ the class of continuous function $\varphi$ on the torus having Fourier series of the form

$$
\varphi(X) \sim b+\sum_{S} b(N) e^{i N \cdot X} .
$$

$C_{S}$ is the closure, in the topology of uniform convergence, of the set of trigonometric polynomials having the form (13). Therefore $C_{S}$ is an algebra, and in fact is a Banach algebra in the uniform topology. To each maximal ideal $M$ in $C_{S}$ is associated a multiplicative linear functional of norm one on $C_{S}$. The value of this functional on $\varphi$ is denoted by $\hat{\varphi}(M)$. The spectrum of $\varphi$ is the closed set of complex numbers $\hat{\varphi}(M)$, where $M$ ranges over the space of maximal ideals. If $F$ is an analytic function defined on a region of the plane containing the spectrum of $\varphi$ then there is an element $\psi$ in $C_{S}$ such that

$$
\hat{\phi}(M)=F[\hat{\varphi}(M)]
$$

we may write simply $\psi=F(\psi)$.

Suppose $\varphi$ is in $C_{S}$ and $\Re \varphi(X) \geqq \varepsilon>0$ for all $X$. We assert that $\Re \hat{\varphi}(M) \geqq \varepsilon$ for all $M$. (The second statement contains the first, because to each point $X$ there is a maximal ideal $M_{X}$ with $\hat{\varphi}\left(M_{X}\right)=\varphi(X)$ for all $\varphi$.) Indeed, the linear functional associated with any $M$ can be extended to the space of all continuous functions without increasing its bound, and so has a representation 


$$
\hat{\varphi}(M)=\int \varphi(X) d \mu_{M}(X)
$$

with

$$
\int\left|d \mu_{M}(X)\right|=1
$$

The function $\varphi_{0}$ constantly equal to one is in $C_{S}$ and its value in every maximal ideal is one. From (14) we obtain

$$
1=\hat{\varphi}_{0}(M)=\int d \mu_{M}(X) .
$$

It follows from (15) and (16) together that $\mu_{M}$ is a non-negative measure. Therefore in (14) we can separate real and imaginary parts to obtain

$$
\Re \hat{\varphi}(M)=\int \Re \varphi(X) d \mu_{M}(X) \geqq \varepsilon
$$

as was to be proved.

Let $p$ be an arbitrary fixed positive number. Suppose $\varphi$ is in $C_{S}$ and $\Re \varphi(X) \geqq \varepsilon>0$. By what has just been proved, the function $z^{p}$ is analytic on the spectrum of $\varphi$, so we can form $\varphi^{\prime}$ in $\mathrm{C}_{S}$ satisfying

$$
\hat{\varphi}^{p}(M)=\hat{\varphi}(M)^{p}
$$

Two cases of (17) are important. First, if $M_{X}$ is the maximal ideal determined by a point $X$ of the torus then (17) becomes

$$
\varphi^{p}(X)=\varphi(X)^{p}
$$

Second, there is a distinguished maximal ideal $M_{0}$ such that ${ }^{1}$

$$
\hat{\varphi}\left(M_{0}\right)=\int \varphi(X) d \sigma(X) .
$$

We conclude from (17) that

$$
\int \varphi^{p}(X) d \sigma(X)=\left(\int \varphi(X) d \sigma(X)\right)^{p} .
$$

The second property of half-spaces has not been used up to this point. We appeal to it now in order to observe that every real trigonometric polynomial is the real part of a trigonometric polynomial in $C_{S}$.

We are ready to give the proof of Theorem 2. Suppose first that $f$ is a positive trigonometric polynomial:

$$
f(X)=\sum b(N) e^{i n \cdot X} \geqq \varepsilon>0
$$

$1 d \sigma(X)$ denotes the element of Haar measure on the torus normalized to have unit total mass. 
and set

$$
\varphi(X)=b(0)+2 \sum_{S} b(N) e^{i N \cdot X} .
$$

Then $\varphi$ belongs to $C_{S}$, and we can write $\varphi=f+i g$ where $g$ is a real trigonometric polynomial having mean value zero. The expression for $\varphi$ in polar form is

$$
\varphi=w(\cos \alpha+i \sin \alpha),
$$

where $w(x)=|\varphi(X)|$ and $-\pi / 2<\alpha(X)<\pi / 2$.

Making use of (18) and (19) and the notation just introduced we have

$$
\begin{aligned}
\left(\int f d \sigma\right)^{p} & =\left(\int \varphi d \sigma\right)^{p}=\int \varphi^{p} d \sigma=\int w^{p}(\cos p \alpha+i \sin p \alpha) d \sigma \\
& =\int w^{p} \cos p \alpha d \sigma \geqq \cos \frac{p \pi}{2} \int\left(f^{2}+g^{2}\right)^{p / 2} d \sigma \geqq \cos \frac{p \pi}{2} \int|g|^{p} d \sigma .
\end{aligned}
$$

That is, with $A=\left(\cos \begin{array}{c}p \pi \\ 2\end{array}\right)^{-1 / p}$,

$$
\left(\int|g|^{p} d \sigma\right)^{i / p} \leqq A \int f d \sigma \text {. }
$$

From this inequality it is simple to prove (12) with a certain constant $B$ in place of $K_{p}$. By continuity the result holds for trigonometric polynomials which are non-negative but not necessarily bounded from zero.

The passage to trigonometric polynomials of arbitrary sign is not quite trivial. If $f$ is real and has the form

$$
f=g-h
$$

where $g$ and $h$ are non-negative trigonometric polynomials, then by what we have proved

$$
\left\|T_{S} f\right\|_{p}^{p} \leqq\left\|T_{S} g\right\|_{p}^{p}+\left\|T_{S} h\right\|_{p}^{p} \leqq B^{p}\left[\|g\|_{i}^{p}+\|h\|_{1}^{p}\right] .
$$

Decompose $f$ into its positive and negative parts :

$$
f=f_{+}-f_{-} ; f_{+}, f_{-} \geqq 0, f_{+} \cdot f_{-} \equiv 0 .
$$

If $f_{+}$and $f_{-}$were trigonometric polynomials we could choose them for $g$ and $h$ and obtain from (20)

$$
\left\|T_{S} f\right\|_{p}^{p} \leqq B^{p}\left[2\|f\|_{1}^{p}\right] .
$$

Of course they are never trigonometric polynomials unless one of them vanishes, but they are non-negative continuous functions, and so can be 
approximated uniformly by non-negative trigonometric polynomials, say by $g_{n}$ and $h_{n}$ respectively. Set $f_{n}=g_{n}-h_{n}$. Writing (20) with $f_{n}, g_{n}$, and $h_{n}$ and passing to the limit we obtain

$$
\lim \left\|T_{S} f_{n}\right\|_{p}^{p} \leqq 2 B^{p}\|f\|_{1}^{p} .
$$

But the sequence $T_{S} f_{n}$ obviously tends to $T_{S} f$ in the norm of $L^{2}$, and so in $L^{p}$ for each $p<2$, so we obtain (21) after all.

There is no difficulty in extending the result to arbitrary complex trigonometric polynomials, with a new constant which we call $K_{p}$, and so the theorem is proved.

CoRollary. If $S$ is a half-space in $\mathscr{L}_{k}$ or in $\mathscr{L}$, the operation $T_{S}$ transforms $L$ into $L^{p}$ for each $p<1$ in the following sense: every Fourier series is carried by $T_{S}$ into a series summable in the metric of $L^{p}$ to a limit function. The summation is effected by every approximate identity consisting of trigonometric polynomials. The transformation carrying $f$ into $T_{S}$ satisfies (12).

Let $f$ be an arbitrary summable function on the torus, and let $\left\{e_{1}, e_{2}, \cdots\right\}$ be an approximate identity consisting of trigonometric polynomials. (That is, each $e_{j}$ is a non-negative trigonometric polynomial with mean value one and $e_{j} * f$ tends to $f$ in the norm of $L$ for each $f$.) Then $\left\{e_{j} * f\right\}$ is a Cauchy sequence in $L$ consisting of trigonometric polynomials. By Theorem 2, $\left\{T_{s}\left(e_{j} * f\right\}\right.$ is a Cauchy sequence in $L^{p}$ for $p<1$. Consequently $T_{S}\left(e_{j} * f\right)$ converges in the metric of $L^{p}$ to a limit function $T_{S} f$, and (12) clearly holds. This is just the statement of the Corollary.

We do not know whether any method of summation effects pointwise convergence almost everywhere of the series for $T_{S} f$. However the Corollary shows that $T_{S} f$ always exists as a limit in mean. If $f$ is a real summable function, its conjugate can be defined as the real function $g$ such that $f+i g=T_{S} f$. Then $g$ exists as a limit in mean, and has many of the properties one expects of a conjugate function in one variable. Our proofs have made strong use of the fact that $S$ is a half-space; we do not know whether $T_{S} f$ exists in any sense whatever if $S$ is, for example, the first quadrant of $\mathscr{L}_{2}$.

The device used to prove Theorem 2 can be used to extend other classical theorems about Fourier series in one variable.

It is possible to assert the conclusion of Theorem 2 for certain sets $S$ which are not quite half-spaces. For simplicity consider a half-space in $\mathscr{L}_{2}$. It consists exactly of those lattice-points $(m, n)$ satisfying

$$
m \alpha+n \beta>0
$$

for some irrationally related numbers $\alpha$ and $\beta$; or else $S$ consists of the 
lattice points satisfying (22) for some rationally related $\alpha$ and $\beta$, together with non-zero points on one ray from the origin of the line

$$
m \alpha+n \beta=0
$$

We shall consider a half-space $S$ of the second type. Denote the other ray (including the origin), which is not contained in $S$, by $R$. With the use of the theorem on conjugate functions of one variable it is easy to prove that Theorem 2 holds for the set $R$ in place of $S$. It follows that the augmented half-space $H$ consisting of all $(m, n)$ with $m \alpha+n \beta \geqq 0$ has the same property.

In the same way we can add to a half-space of dimension $k$ in $\mathscr{L}_{k}$ disjoint half-spaces of lower dimension, obtaining new sets for which Theorem 2 and its corollary hold.

Let $R$ and $S$ be two half-spaces, or more generally, any sets having the property of Theorem 2 . Then $T_{R}-T_{S}$ is an operator carrying $L$ into $L^{p}$ for each $p<1$. For example, let $R$ and $S$ be the modified half-spaces in $\mathscr{L}_{2}$ defined respectively by $m \geqq 0$ and $n<0 . \quad T_{R}-T_{S}$ operates on trigonometric series in two variables by multiplying each term by a factor $\varepsilon_{m n}$; this factor is 1 in the first quadrant (including the boundary) and -1 in the third quadrant (excluding the boundary), and vanishes in the second and fourth quadrants.

By Bochner's theorem the operator $T_{R}-T_{S}$ carries $L^{p}$ into itself for $p>1$. It is interesting to compare these results with theorems of similar nature but different proof by Caldéron and Zygmund [3].

\section{A generalization of Paley's theorem.}

THeOREm 3. Let $S$ be a half-space in $\mathscr{L}_{k}$. Suppose $\{w(N)\}$ is a set of non-negative numbers defined for $N$ in $S$, and having the property that

$$
|b|+\sum_{S}|b(N)| w(N)<\infty
$$

whenever (13) is the Fourier series of a function in $C_{S}$. Then

$$
\sum_{S} w(N)^{2}<\infty
$$

Proof. As for one variable, the hypothesis implies that

$$
\sum_{S} w(N) e^{i \varphi(N)} e^{i N \cdot X}
$$

is the image under $T_{S}$ of some Fourier-Stieltjes series, no matter how the real numbers $\varphi(N)$ are chosen. This assertion remains true if any of the $w(N)$ are replaced by zero, because (23) continues to hold. Suppose now (24) is false. Then at least one of the $2^{k}$ congruent cones

$$
\pm n_{1} \geqq 0, \pm n_{2} \geqq 0, \cdots, \pm n_{k} \geqq 0
$$


obtained by choosing the $k$ signs in these inequalities contains points over which the sum (24) diverges. Denote such a cone by $C$. Replace $w(N)$ by zero for all $N$ in $S-C$. Finally, by a linear change of variables bring $C$ into coincidence with the preferred cone

$$
n_{1} \geqq 0, n_{\mathrm{z}} \geqq 0, \cdots, n_{k} \geqq 0 .
$$

(The transformation carries $S$ onto a new half-space.) Now we have a counter-example to the theorem (which is assumed to be false) in which $w(N)$ vanishes for all $N$ not in $C$.

Extend $w$ to the complement of $S$ so that

$$
\sum w(N) e^{i \varphi(N)} e^{i N \cdot X}
$$

is the Fourier-Stieltjes series of a measure $\mu$. For $0<r<1$ define a function $f_{r}$ on the torus by the absolutely convergent series

$$
\sum w(N) r^{\left|n_{1}\right|+\cdots+\mid n_{k} !} e^{i \varphi(N)} e^{i N \cdot X} .
$$

For each $r$ we have $\left\|f_{r}\right\|_{1} \leqq \int|d \mu|$, and so by the corollary to Theorem 2

$$
\left\|T_{S} f_{r}\right\|_{p} \leqq K_{p}\left\|f_{r}\right\|_{1} \leqq K \quad(0<r<1) .
$$

Moreover the corollary implies that

$$
T_{S} f_{r}(X)=\sum_{S} w(N) r^{\left|n_{1}\right|+\cdots+\left|n_{k}\right|} e^{i \varphi(N)} e^{i V^{*} \cdot X} .
$$

Since $w(N)$ vanishes on $S-C$ this can be written

$$
T_{S} f_{r}(X)=\sum_{C} w(N) r^{n_{1}+\cdots+n_{k}} e^{i \varphi(N)} e^{i N \cdot X} .
$$

On account of (26) this series belongs to the space $H^{n}(p<1)$, whose elements are analytic functions of $k$ variables. A theorem of Zygmund $[6$, p. 208] asserts that

$$
\lim _{r \uparrow 1} T_{S} f_{r}(X)
$$

exists almost everywhere on the torus. But the numbers $\varphi(N)$ are arbitrary, and so we conclude as in the case of one variable that

$$
\sum_{e} w(N)^{2}<\infty .
$$

This contradiction shows that the theorem is true.

5. A counter-example. Let $S$ be the subset of $\mathscr{P}$ consisting of all lattice points $N=\left(n_{1}, \cdots\right)$ with each $n_{j} \geqq 0$. ( $S$ is not a half-space, but rather the infinite-dimensional analogue of the quadrant in $\mathscr{L}_{2}$.) Denote by $\Lambda$ the subset of $S$ on which $\sum n_{j}=1$. 
Theorem (Bohr [2, p. 468]). For each $\varphi$ in $C_{S}$ with Fourier series (11) we have

$$
\sum_{\Lambda}|a(N)| \leqq\|\varphi\|_{\infty}
$$

This result states exactly that the sequence $\{w(N)\}$ equal to 1 on $\Lambda$ and 0 on $S-\Lambda$ has the property of Paley's theorem; but $w(N)$ does not even tend to zero, and certainly is not square-summable. So Paley's theorem fails decisively in this setting.

For this sequence the series (25) is

$$
\sum_{j=1}^{\infty} e^{i t} j e^{i x_{j}},
$$

and this is the image under $T_{S}$ of a Fourier-Stieltjes series no matter how the real numbers $t_{j}$ are chosen. For appropriate values of $t_{1}, t_{2}$, $\cdots(27)$ is non-summable for almost every point $\left(x_{1}, x_{2}, \cdots\right)$, and this is true for each Toeplitz method of summation. Hence the theorem on the summability of conjugate series in one variable cannot be extended to this extreme situation. The analogous problem in two variables is open, so far as we know, both for half-spaces and for quadrants.

\section{REFERENCES}

1. S. Bochner, Additive set functions on groups, Ann. of Math., 40 (1939), 769-799.

2. H. Bohr. Über die Bedeutung der Potenzreihen unendlich vieler Variabeln in der Theorie der Dirichletschen Reihen $\sum a_{n} / n^{s}$, Nachr. Akad. Wiss. Göttingen, Math.-Phys. Kla., (1913), 441-488.

3. A. P. Caldéron and A. Zygmund, Singular integrals and periodic funciions, Stud. Math. 14 (1954), 249-271

4. H. Helson and D. Lowdenslager, Prediction theory and Fourier series in several variables, to appear in Acta Math.

5. R. E. A. C. Paley, A note on power series, J. London Math. Soc,. 7 (1932), 122-130.

6. A. Zygmund, On the boundary values of functions of several complex variables, I, Fund. Math., 36 (1949), 207-235.

7. _- - Trigonometric Series, Warsaw Lwów, 1935

UNIVERSITY OF CALIFORNIA AT BERKELEY 


\section{PACIFIC JOURNAL OF MATHEMATICS}

\section{EDITORS}

David Gilbarg

Stanford University

Stanford, California

R. A. Beaumont

University of Washington

Seattle 5, Washington

\author{
A. L. Whiteman
}

University of Southern California

Los Angeles 7, California

E. G. Straus

University of California

Los Angeles 24, California

\section{ASSOCIATE EDITORS}

\author{
E. F. BECKENBACH \\ C. E. BURGESS \\ M. HALL \\ E. HEWITT
}

\author{
A. HORN \\ V. GANAPATHY IYER \\ R. D. JAMES \\ M. S. KNEBELMAN
}

L. NACHBIN
I. NIVEN
T. G. OSTROM
H. L. ROYDEN

M. M. SCHIFFER

G. SZEKERES

F. WOLF

K. YOSIDA

\section{SUPPORTING INSTITUTIONS}

\author{
UNIVERSITY OF BRITISH COLUMBIA \\ CALIFORNIA INSTITUTE OF TECHNOLOGY \\ UNIVERSITY OF CALIFORNIA \\ MONTANA STATE UNIVERSITY \\ UNIVERSITY OF NEVADA \\ OREGON STATE COLLEGE \\ UNIVERSITY OF OREGON \\ UNIVERSITY OF SOUTHERN CALIFORNIA
}

\author{
STANFORD UNIVERSITY \\ UNIVERSITY OF UTAH \\ WASHINGTON STATE COLLEGE \\ UNIVERSITY OF WASHINGTON \\ AMERICAN MATHEMATICAL SOCIETY \\ CALIFORNIA RESEARCH CORPORATION \\ HUGHES AIRCRAFT COMPANY \\ THE RAMO-WOOLDRIDGE CORPORATION
}

Mathematical papers intended for publication in the Pacific Journal of Mathematics should be typewritten (double spaced), and the author should keep a complete copy. Manuscripts may be sent to any of the editors. All other communications to the editors should be addressed to the managing editor, E. G. Straus at the University of California, Los Angeles 24, California.

50 reprints per author of each article are furnished free of charge; additional copies may be obtained at cost in multiples of 50 .

The Pacific Journal of Mathematics is published quarterly, in March, June, September, and December. The price per volume (4 numbers) is $\$ 12.00$; single issues, $\$ 3.50$. Back numbers are available. Special price to individual faculty members of supporting institutions and to individual members of the American Mathematical Society: $\$ 4.00$ per volume; single issues, $\$ 1.25$.

Subscriptions, orders for back numbers, and changes of address should be sent to Pacific Journal of Mathematics, 2120 Oxford Street, Berkeley 4, California.

Printed at Kokusai Bunken Insatsusha (International Academic Printing Co., Ltd.), No. 6, 2-chome, Fujimi-cho, Chiyoda-ku, Tokyo, Japan.

\section{PUBLISHED BY PACIFIC JOURNAL OF MATHEMATICS, A NON-PROFIT CORPORATION}

The Supporting Institutions listed above contribute to the cost of publication of this Journal, but they are not owners or publishers and have no responsibility for its content or policies. 


\section{Pacific Journal of Mathematics}

\section{Vol. 8, No. 3 \\ May, 1958}

Michael Israel Aissen, A set function defined for convex plane domaines... . 383

Robert Ellis, Distal transformation groups ................... 401

Ciprian Foias, On a commutative extension of a commutative Banach algebra ....................................... 407

Jerry William Gaddum, Linear inequalities and quadratic forms ......... 411

Allen A. Goldstein and Elliott Ward Cheney, Jr., A finite algorithm for the solution of consistent linear equations and inequalities and for the Tchebycheff approximation of inconsistent linear equations...........

William L. Hart and T. S. Motzkin, Proof of the fundamental theorem on implicit functions by use of composite gradient corrections .......... 429

Henry Berge Helson, Conjugate series and a theorem of Paley .......... 437

Wu-Chung Hsiang, Abelian groups characterized by their independent subsets....................................... 447

John W. Lamperti, On the isometries of certain function-spaces ........ 459

Karel DeLeeuw and Walter Rudin, Extreme points and extremum problems

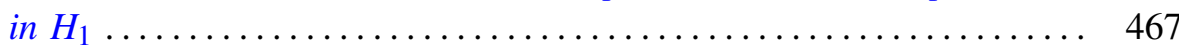

Eugene Lukacs, Some extensions of a theorem of Marcinkiewicz........ 487

George W. Mackey, Multiplicity free representations of finite groups ..... 503

Eben Matlis, Injective modules over Noetherian rings ............. 511

John William Neuberger, Continuous products and nonlinear integral equations

Lawrence Edward Payne and Hans F. Weinberger, New bounds for solutions of second order elliptic partial differential equations...

William T. Reid, A Prüfer transformation for differential systems ........ 575

Howard L. Rolf, The free lattice generated by a set of chains ...

K. M. Saksena, Inversion and representation theorems for a generalized

Laplace integral....................................... 597

Daniel Shanks, Two theorems of Gauss......................... 609

Paul Slepian, On the Lebesgue area of a doubled map ............... 613

Otto Szász and Nelson Paul Yeardley, Jr., The representation of an analytic function by general Laguerre series . ..................... 621

Alan C. Woods, On two-dimensional convex bodies ................. 635 